Int. J. Electrochem. Sci., 14 (2019) 5096 - 5106

\title{
Porous N-doped Carbon Derived from ZIF-8/Reduced Graphene oxide/Polypyrrole Composite for High Performance Supercapacitor
}

\author{
Yin Liu ${ }^{1}$, Qingli Sui ${ }^{1}$,Yongjin Zou ${ }^{1,2}$, Cuili Xiang ${ }^{1, *}$, Fen Xu ${ }^{l}$, Jingjing Xie ${ }^{3}$, Lixian Sun ${ }^{1, *}$ \\ ${ }^{1}$ Guangxi Key Laboratory of Information Materials, Guilin University of Electronic Technology, \\ Guilin 541004, P.R. China \\ ${ }^{2}$ School of Chemistry and Chemical Engineering, Hunan University of Science and Technology, \\ Xiangtan, 411201, China \\ ${ }^{3}$ State Key Laboratory of Advanced Technology for Materials Synthesis and Processing, Wuhan \\ University of Technology, Wuhan, 430070, China \\ *E-mail: xiangcuili@guet.edu.cn, sunlx@guet.edu.cn
}

doi: $10.20964 / 2019.06 .19$

Received: 6 January 2019 / Accepted: 10 March 2019 / Published: 10 May 2019

\begin{abstract}
Porous N-doped carbon nanomaterials have great potential in supercapacitor owing to their high surface area, excellent chemical stability and good hydrophilic property. In this work, ZIF-8 nanocystals were well grown on the surface of polypyrrole/reduced graphene oxide composite (ZIF8/PPy/rGO). After carbonization of ZIF-8/PPy/rGO and chemical etching of the product, a porous carbon with N-doping (PNC) and proper structure was obtained. In comparison with the carbonized ZIF-8 and PPy/rGO, the ZIF-8/PPy/rGO derived carbon possesses much higher electrochemical performance. As electrodes in supercapacitor, the PNC exhibits a significantly enhanced specific capacitance (236 F g$~^{-1}$ at $1 \mathrm{~A} \mathrm{~g} \mathrm{~g}^{-1}$ ), superior rate capability (retained $86.1 \%$ from 1 to $20 \mathrm{~A} \mathrm{~g}^{-1}$ ) and excellent cycling stability (over $96.8 \%$ capacity retained after 10000 cycles), exhibiting its potential for electrode materials with high performance.
\end{abstract}

Keywords: ZIF-8; Supercapacitor; Porous carbon; Graphene; N-doping

\section{FULL TEXT}

(C) 2019 The Authors. Published by ESG (www.electrochemsci.org). This article is an open access article distributed under the terms and conditions of the Creative Commons Attribution license (http://creativecommons.org/licenses/by/4.0/). 